\title{
Interacción entre la extensión y la investigación para el abordaje de los problemas productivos del complejo pesquero en Uruguay
}

Sujetos y relaciones en extensión universitaria

\section{다(1)(2)(2)}

Oscar Galli

ogalli45@gmail.com

(iD) orcid.org/0000-0003-4171-781X

\section{Mariana Mendy}

mariana.mendy@cseam.udelar.edu.uy

(iD) orcid.org/0000-0003-2703-0843
Walter Norbis

wnorbis@fcien.edu.uy

(iD) orcid.org/0000-0002-7819-3450

Universidad de la República, Uruguay.
RECEPCIÓN: 26/06/20

ACEPTACIÓN FINAL: 02/10/20

\section{Resumen}

Este trabajo describe y analiza una experiencia de investigación y extensión con los trabajadores de la Intergremial Marítima de Uruguay, quienes cuestionaban la sustentabilidad del modelo de explotación pesquero industrial. Se emplearon técnicas cualitativas y cuantitativas de relevamiento y análisis de información y participación activa entre actores sociales (investigación participativa). Considerando el complejo pesquero como productivo, se describen los vínculos de los sindicatos con el equipo universitario interdisciplinario y, desde una perspectiva extensionista, la producción conjunta de conocimiento, la apropiación del mismo por los actores y el planteo de propuestas alternativas al modelo de explotación cuestionado. Se compatibilizaron los tiempos de investigación con las urgencias de los actores sociales que demandaron la intervención. A través de la identificación de problemas críticos, el proceso de extensión permitió establecer propuestas de cambio al modelo de explotación y el desafío de construir agenda de investigación a partir de la actividad de extensión.
Interaction between the extension and the research involved in approaching the fishing complex productivity problems in Uruguay

\section{Abstract}

The objective of this work was describing and analyzing a research and extension experience with the workers of the Intergremial Marítima of Uruguay, who questioned the sustainability of the industrial fishing exploitation model.

Qualitative and quantitative techniques were used for data collection and analysis, including active participation among social actors (participatory research). Considering the fishing complex as a productive complex, the bonds of the syndicate with the interdisciplinary university team are described from an extensive perspective. This includes, the coproduction of knowledge, the appropriation of it by the actors and the alternative proposals to the exploitation model that has been questioned. The research time was reconciled with the urgencies of the social actors who demanded the intervention. From identifying critical issues, the extension process allowed us to establish proposals for changes to the exploitation model and the challenge of building a research agenda from the extension activity.

Keywords: fishing; fishing complex; Uruguay; university extension; investigation.
Interação entre extensão e pesquisa para uma abordagem aos problemas produtivos do complexo pesqueiro no Uruguai

\section{Resumo}

O objetivo deste trabalho foi descrever e analisar uma experiência de pesquisa e extensão com os trabalhadores da Intergremial Marítima do Uruguai, que questionavam a sustentabilidade do modelo de exploração da pesca industrial. Técnicas qualitativas e quantitativas foram utilizadas para levantar e analisar informações e a participação ativa entre os atores sociais (pesquisa participativa). Considerando o complexo pesqueiro produtivo, descrevem-se os vínculos dos sindicatos com a equipe interdisciplinar universitária e, numa perspectiva de extensão, a produção conjunta do conhecimento, a apropriação dele pelos atores e a formulação de propostas alternativas ao modelo de exploração questionado. Os tempos de pesquisa foram conciliados com as urgências dos atores sociais que exigiam a intervenção. A partir da identificação de problemas críticos, o processo de extensão possibilitou o estabelecimento de propostas de mudanças no modelo de exploração e o desafio de construir uma agenda de pesquisa baseada na atividade extensionista.

Palavras-chave: pescaria, complexo de pesca Uruguai; extensão universitária; pesquisa. 


\section{Introducción}

Este artículo tiene como objetivo describir y analizar una experiencia de investigación y extensión desarrollada por un equipo interdisciplinario formado por docentes de diferentes servicios de la Universidad de la República. Esta experiencia constituyó un intento de respuesta a la demanda planteada por los trabajadores sindicalizados del sector pesquero organizados en tres sindicatos de la Intergremial Marítima (Trabajadores del Mar, ${ }^{1}$ Patrones de Pesca $^{2}$ y Maquinistas Navales).

La demanda tuvo su origen en la necesidad de plantear soluciones que permitieran resolver problemas productivos que cuestionaban la sustentabilidad del modelo de explotación de la pesca industrial marítima en Uruguay y aportar elementos que contribuyan al diseño social de un modelo productivo pesquero superador del actual. En este contexto, se elaboró el proyecto "Abordaje de los Problemas Productivos del Complejo Pesquero Uruguayo", en conjunto con los sindicatos, los cuales plantearon como hipótesis de trabajo que el modelo pesquero estaba agotado. La depredación de los recursos pesqueros, la desigualdad social, las malas condiciones de trabajo y de la flota pesquera industrial, así como el acceso restringido de la sociedad uruguaya a un alimento de alto valor nutricional fueron los principales indicadores que fundamentaron esta hipótesis y, de confirmarse la misma, se contempló la posibilidad de construir un modelo productivo pesquero alternativo.

Este proyecto significó un desafío teórico y metodológico debido a la necesidad de abordarlo de forma multidimensional, para lo cual se utilizó como referencia el concepto de complejo productivo, empleado en el estudio de los sectores agroindustriales (Vigorito, 1978; Scarlato, 1994). Un complejo productivo es un espacio económico en el que la reproducción y transformación son fuertemente interdependientes; o sea, en el que los componentes se relacionan estrechamente entre sí, ya sea por factores internos o ubicados en el medioambiente en que se sitúa el complejo. La relación mencionada se debe a que forma parte de a) una secuencia técnica de tipo recursos/producción primaria/comercialización/procesamiento/distribución y b) una estructura de propiedad (Buxedas, 1994).

En el caso del complejo pesquero, Mendy et al. (2011) señalan:

"El complejo pesquero nacional abarca múltiples aspectos relacionados entre sí y susceptibles de ser abordados desde diversos saberes. Pero de la misma forma que en la realidad, el sector constituye un complejo que encadena diversas dimensiones, su abordaje no puede ser realizado en forma compartimentada desde distintas áreas de conocimiento (...), necesariamente requiere un abordaje integrador. El primer desafío, por tanto, es cómo abordar este 'objeto de estudio' complejo y multidimensional sin que el resultado sea una sumatoria de capítulos escritos, cada uno desde una mirada disciplinar. (...) Se considera que un complejo productivo es un entramado de relaciones, estructuras y procesos que es necesario aprender dando cuenta de su movimiento, su dinámica y sus contradicciones". (p. 3)

1) El Sindicato Único de Trabajadores del Mar y Afines nuclea a los marineros de pesca industrial y pesca artesanal así como a los trabajadores de las plantas pesqueras industriales.

2) Los Patrones de Pesca son los trabajadores que cumplen las tareas de capitán de la embarcación en el caso de la flota industrial. 
La Pesca Industrial Marítima en Uruguay está compuesta por diversas flotas de barcos que tienen diferentes especies objetivo. La flota industrial de altura realiza sus actividades pesqueras con redes de arrastre de fondo dentro de la Zona Común de Pesca ArgentinoUruguaya (ZCPAU), entre los 50 y 400 metros de profundidad, siendo la especie objetivo la merluza (Merluccius hubbsi). La flota industrial costera efectúa sus actividades dentro de la ZCPAU con redes de arrastre de fondo, a profundidades menores de 50 metros, siendo la especie objetivo la corvina (Micropogonias furnieri). Estas dos flotas sustentan las principales pesquerías industriales en Uruguay. También en aguas uruguayas y dentro de la ZCPAU se desarrollan otras pesquerías con barcos industriales que hacen sus actividades con artes de pesca diferentes del arrastre de fondo y que no tienen como especies objetivo ni merluza ni corvina (Etchebehere et al., 2018).

La pesca artesanal comprende a las embarcaciones que no pueden superar las 10 toneladas de registro bruto (TRB) y que utilice las artes de pesca que DINARA establezca para cada zona (Ley de Pesca № 19175). Se desarrolla a lo largo de todo el territorio uruguayo (ver localización Figura 1), tanto en aguas marinas y del Río de la Plata como en lagunas y cursos de agua continentales (Etchebehere et al., 2018).

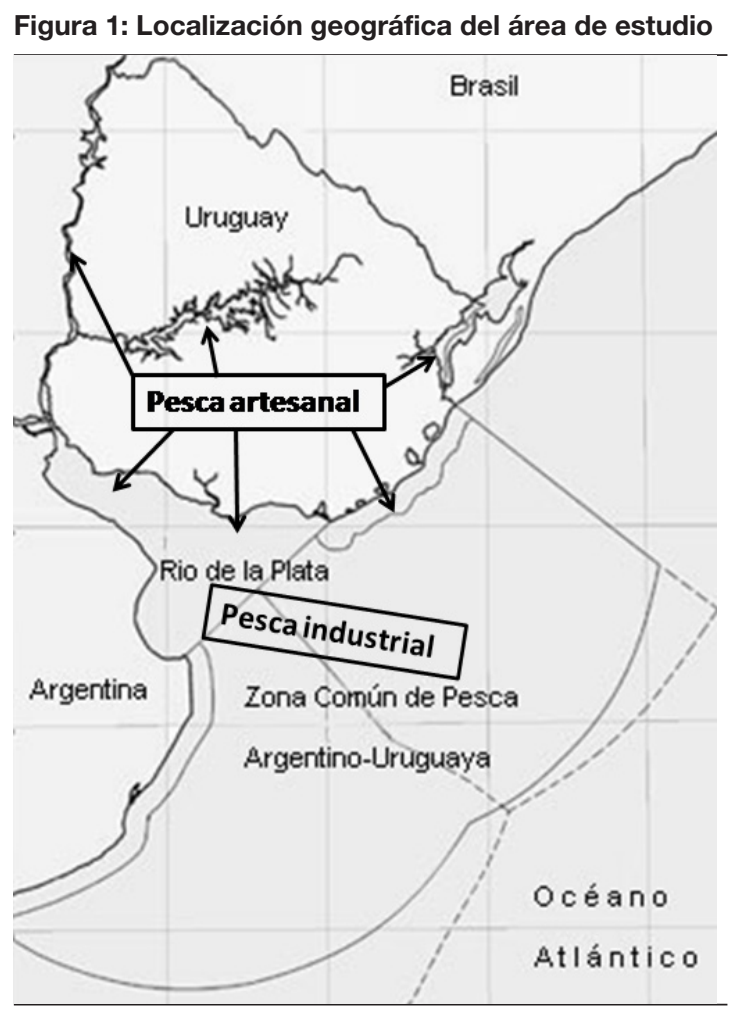

Fuente: Avdalov, Pereira (2015). La pesca, el pescado y la alimentación. MGAP-DINARA-INFOPESCA. Modificado por los autores.

El proyecto se desarrolló entre los años 2011 y 2013 y el principal resultado estuvo vinculado a la confirmación de la hipótesis inicial: el modelo pesquero se encontraba agotado en todas sus dimensiones, ecológica, comercial, productiva, social y tecnológica. 
Culminado el proyecto, entre 2013 y 2019 se continuó el trabajo bajo un enfoque interdisciplinario y este proceso posterior permitió desarrollar algunas reflexiones sobre la pertinencia de los resultados del proyecto y la apropiación del mismo por parte de los actores a los efectos de orientar su acción colectiva.

En este trabajo se describe cómo se vincularon los sindicatos de trabajadores del mar con un equipo universitario, lo cual dio origen al proyecto mencionado, y al mismo tiempo se discute desde una perspectiva extensionista la producción de conocimiento con los actores sociales que intervienen en la investigación. A continuación, se describe y discute asimismo el abordaje metodológico, el que estuvo basado en un enfoque de investigación participativa. Seguidamente, se presentan los resultados obtenidos mediante el desarrollo de esta experiencia: la apropiación por los actores sociales del conocimiento generado a partir de distintas instancias formativas y de discusión, lo cual habilitó propuestas alternativas al modelo agotado. A modo de conclusión, se enfatiza sobre la importancia del vínculo de la universidad con la sociedad, en especial con el sector productivo, y se plantea un desafío que tiene que ver con la posibilidad de elaborar una agenda de investigación a partir de la actividad de extensión.

\section{La historia de una vinculación y el nacimiento del proyecto}

Los primeros contactos con los trabajadores se dieron en el año 2008. En ese momento, el planteo no pasaba de una inquietud acerca del futuro del sector especialmente en vinculación con las fuentes de empleo de sus trabajadores debido a la disminución de las capturas y como consecuencia de la flota pesquera. A posteriori, y en función de una serie de reuniones entre el equipo universitario de la Unidad de Relacionamiento con el Sector Productivo (URSP-SCEAM) $)^{3}$ y la Intergremial Marítima a iniciativa del SUNTMA ${ }^{4}$, comenzó a tomar forma la idea de diseñar un proyecto que construyera conocimientos actualizados y proporcionara insumos para el planteo de soluciones a los problemas productivos que aparentemente estaban en la base de la crisis de sustentabilidad del modelo de explotación de la pesca industrial. La demanda de los trabajadores partió de un diagnóstico basado en su experiencia y conocimiento tácito más la evidencias señaladas en trabajos científicos previos (Galli, 2005, 2007).

El comienzo fue un proceso de extensión en el que estaban presentes los elementos definitorios de esta función: la vinculación entre la universidad y la sociedad, vinculación a partir de un problema que la comunidad no puede resolver por sí misma, existencia de un proceso bidireccional (diálogo), y vocación de cambio para superar el problema identificado (Tünnermann, 2000; Fresán, 2004). El proceso inicial fue de construcción de demanda y tuvo como propósito, precisamente, la construcción colectiva del problema de investigación y los objetivos centrales a lograr para elaborar una síntesis superadora entre la demanda del

3) La Unidad de Relacionamiento con el Sector Productivo (URSP), actualmente Área Sector Productivo y Organizaciones Sociales, integra la Unidad Académica del Servicio Central de Extensión y Actividades en el Medio (SCEAM) de la Universidad de la República.

4) La Intergremial Marítima es un espacio de coordinación de los tres sindicatos que nuclean a los trabajadores del mar: Sindicato Único de Trabajadores del Mar y Afines (SUNTMA), Sindicato Único de Patrones de Pesca del Uruguay (SUDEPPU) y Centro de Maquinistas Navales (CMN). 
colectivo social y el problema definido a priori por los equipos universitarios. El proceso de investigación fue, por lo tanto, resultado de la conjunción entre las demandas e inquietudes de los trabajadores y un equipo académico con interés en desarrollar investigación en el sector pesquero. El equipo académico, de carácter interdisciplinario, se fue conformando en paralelo al proceso de construcción de la demanda. Al equipo original, compuesto por economistas y sociólogos, se incorporaron biólogos pesqueros, psicólogos y trabajadores sociales.

Históricamente, los sindicatos de la pesca, y especialmente el SUNTMA, han planteado la existencia de una crisis estructural de base productiva en el complejo pesquero vinculada al modelo de explotación extractivo-exportador. El colectivo sindical (especialmente el sector de trabajadores de la pesca industrial) ubicó los síntomas de esta crisis en diversas dimensiones (social, económica, ambiental y tecnológica), identificadas a partir de su experiencia laboral en el sector. En conjunto con la constatación primaria de manifestaciones de la crisis, señalaron la necesidad de una intervención del sector académico para desarrollar un diagnóstico y análisis más complejo, que proporcionara fundamento para el planteo de propuestas alternativas.

A partir de la conformación del equipo de trabajo se diseñó el proyecto de investigación que estableció la caracterización del complejo pesquero uruguayo mediante la identificación de sus problemas y nudos críticos, de forma tal de posibilitar el diseño de propuestas orientadas a superarlos. El segundo aspecto que el proyecto planteó fue la necesidad de contribuir a valorizar el conocimiento y la capacidad de propuesta de los actores organizados a través de un proceso de co-construcción de conocimiento. Estos objetivos tuvieron origen en el proceso de extensión que permitió una construcción de demanda que evidenció un importante acervo de conocimientos en el colectivo de trabajadores y su necesidad de participación en todas las etapas del proyecto, trascendiendo la concepción de investigar "para" hacia una concepción de investigar "con".

Era necesario abordar el complejo pesquero en su totalidad, desde un proceso de investigación que proporcionara un panorama general de sus características y problemas principales. La integración de los actores sociales del sector fue la expresión del trabajo extensionista previo, trabajo no exento de dificultades y que vale la pena analizar a la luz del tiempo transcurrido y de lo desarrollado desde la culminación de la investigación.

El proyecto de investigación buscó trascender la mera observación, descripción y análisis del complejo pesquero, para constituirse en una herramienta que permitiera al colectivo actuar e incidir con mayor eficacia en su medio. De ahí la importancia del trabajo conjunto con el actor sindical, no tomándolo como simple "objeto de estudio" sino como coautor del proceso, componente esencial del proyecto, entendido como proyecto de investigación y de extensión.

\section{La coproducción de conocimientos en los proyectos extensionistas}

Producir conocimiento junto a los actores implica un esfuerzo por construir miradas comunes a partir de las cuales el conocimiento elaborado constituya una síntesis superadora de las especificidades disciplinares y que incorpore el conocimiento de los actores al desarrollo de la investigación. Esta co-construcción abarca dos dimensiones: la interdisciplina (entendida como la conjunción y diálogo de saberes disciplinares especializados) y la transdisciplina (entendida como la conjunción y el diálogo entre saberes disciplinares y saberes populares). 
Interdisciplina porque el problema denominado "crisis del modelo de explotación pesquero" no es solamente económico social, tecnológico o biológico, es de carácter estructural. Se presenta en relaciones, estructuras y procesos y en diferentes dimensiones (social, tecnológica, biológica, económica, etc.), por lo que se requiere del concurso de varias disciplinas para abordarlo en su complejidad (Mendy et al., 2013).

Desde el punto de vista de la interdisciplina, este abordaje no se resolvía con la formación de un equipo de universitarios de diferentes áreas del conocimiento. Eso consistía en el primer paso. Lo sustancial era poner en diálogo a las formaciones disciplinares, lo cual en la práctica no fue nada fácil. La fragmentación disciplinar y profesional, entre otros aspectos, trata de metodologías, marcos teóricos y lenguajes diferentes. ¿Cómo superar las diferencias? Para ello se diseñaron las denominadas "instancias internas de formación", pensadas como espacios en los que los integrantes del grupo de investigadores pertenecientes a una disciplina preparaban una exposición vinculada al sector pesquero y la exponían a los demás. Estos espacios permitieron una base de lenguaje y comprensión común, de saber "de qué habla el otro cuando habla de...", ubicando las diferentes ideas en un marco lógico (más rico y más complejo) que trascendiera los marcos disciplinarios propios.

Desde la perspectiva de la transdisciplina, se trata de contar con el conocimiento y la interpretación de la realidad de los actores no universitarios, portadores de conocimiento "no-académico" pero útil para desentrañar la realidad a explicar y elaborar propuestas superadoras. Para ello fue necesario diseñar dispositivos que crearan condiciones para poner ambos conocimientos en diálogo. Por ejemplo: en la implementación de talleres sobre organización del trabajo, el equipo identificó la dificultad de comprensión de los lenguajes entre los participantes del proyecto. A partir de allí, y junto a un equipo de 30 trabajadores, se evaluó el cambio de metodología y se resolvió desarrollar un taller conducido por trabajadores para que explicaran temas que, siendo básicos para ellos, eran de difícil comprensión para el equipo de investigación. En estas instancias los trabajadores fueron explicando sus procesos de trabajo de forma tal de construir un lenguaje común en el equipo. El abordaje de lo inter y transdisciplinario implicó la construcción de dispositivos y espacios de diálogo que permitieran conocer y reconocer los marcos conceptuales de los integrantes, evaluar su pertinencia y generar síntesis superadoras (Etchebehere et al., 2018).

Los participantes del proyecto eran portadores de diferentes saberes: el equipo universitario poseedor de un saber académico o "experto" cimentado en una metodología; los trabajadores del mar poseedores de un saber "tácito", ${ }^{5}$ popular, cimentado en la reflexión acerca de sus experiencias. La interacción entre estos conocimientos constituyó un factor clave en el momento de pensar qué técnicas concretas emplear para relevar, seleccionar y analizar la información. Este diálogo de saberes fue condición necesaria, teniendo en cuenta la perspectiva de investigación orientada a la acción con la que se encaró el trabajo.

5) El conocimiento tácito es aquel desarrollado a través de la observación, experiencias, ejemplos y orientaciones de pares. Si bien puede ser desarrollado en todos los contextos, el conocimiento vinculado a los procesos de trabajo es un ejemplo típico. Michel Polanyi (1966) es pionero en la definición de este concepto que es empleado en múltiples análisis de realidades laborales. Una buena síntesis del desarrollo conceptual sobre conocimiento tácito puede encontrarse en Caussin et al. (2019). 
Partir de la hipótesis de "modelo productivo agotado" suponía cuestionar la modalidad de explotación pesquera hegemónica. Sin embargo, es factible que desde el saber académico puedan plantearse preguntas situadas en el actual modelo pesquero, por ejemplo: ¿es necesario desarrollar acciones científicas y evaluaciones para mantener el límite aceptable de explotación de los recursos pesqueros? En un contexto de peligro de sobreexplotación de recursos, la respuesta afirmativa es obvia. No obstante, también es cierto que los modelos de hacer ciencia pueden ser funcionales a los intereses hegemónicos en la explotación de un recurso, en este caso, a los grupos económicos que se ven beneficiados por la situación y el contexto. Si no se sitúa el conocimiento como "parte" en las relaciones sociales de producción se puede caer en la trampa de la "neutralidad de la ciencia", sin que exista una apropiación crítica del saber académico.

En un modelo contradictorio, con intereses en pugna, son posibles los desencuentros entre conocimientos académicos.

Esta apuesta a la construcción de conocimiento complementando saberes es vehiculizada a partir de la instauración de un funcionamiento horizontal en el equipo de trabajo que, más allá de jerarquías académicas o responsabilidad en la tarea, genere condiciones de posibilidad para un ejercicio democrático de la capacidad de exponer, discutir y contraponer ideas y pareceres. En este sentido, los trabajadores de la pesca, poseedores de conocimientos y experiencias particulares, "corrían con ventaja" respecto del resto del equipo. No partían de disciplinas ni temas sino de problemas y de propuestas. Estas últimas fueron las hipótesis que permitieron generar condiciones para proponer alternativas al modelo productivo actual.

Cabe la mención a una dificultad no menor. La co-construcción de conocimiento no puede ser interpretada en forma idílica. La horizontalidad entre los participantes del proceso no puede ser un espejismo de falsa igualdad que empobrezca los procesos de investigación y extensión. Como señalan Mendy y Marrero (2020):

"la horizontalidad no es un proceso que se pueda construir a partir de la enunciación, pues declarar la igualdad de los actores no anula el devenir histórico que hace que los trabajadores y los académicos estén en posiciones diferentes en cuanto a su capacidad de construir conocimiento. Por tanto, superar las desigualdades implica, en primer lugar, asumirlas y, en segundo lugar, desarrollar procesos que permitan que la horizontalidad sea más que una declaración, la construcción de nuevas condiciones para conocer. Se trata entonces de poner el conocimiento en acción. De este modo, se retoma la propuesta de Fals Borda (1978) del vínculo de lo vivencial con lo racional a partir de la movilización de saberes hacia la identificación y resolución de problemáticas sociales. La validación de este nuevo tipo de conocimiento producido en tales circunstancias será confrontada con la práctica concreta, por parte de los propios productores. Este conocimiento en acción implica también una toma de posición no solo epistemológica sino también ético - política con relación al compromiso con la lucha de los trabajadores y el campo popular". (p. 35)

Es a partir de estas consideraciones (previas al proyecto y posteriores a él, pues el proceso científico-académico no se detiene en el último día del cronograma del proyecto) que se diseñó una metodología que puede ser evaluada en un doble movimiento: su adecuación en el desarrollo del proceso y su evaluación una vez que el proceso culminó. 


\section{El abordaje metodológico}

La metodología de trabajo que se propuso estuvo basada en un enfoque de investigación participativa, en términos de Sirvent (2008, p. 77): "estrategias mediante las cuales la población participa activamente en la toma de decisiones y en la ejecución de una o más fases de un proceso de investigación". Diseñar una investigación de este tipo presenta el desafío de buscar una práctica que sea coherente con lo enunciado en el apartado anterior. La perspectiva de investigación asumió una intencionalidad política: situarse en una posición crítica respecto del modelo primario exportador característico de la matriz productiva nacional y de la producción pesquera en particular (Astori y Buxedas, 1987; Etchebehere et al., 2018). Para ello se tomó en cuenta la perspectiva de los trabajadores, es decir, aquella de la clase subalterna en el sistema de relaciones sociales de producción. En este sentido, Sirvent (2008) afirma:

"Son intentos de búsqueda de una práctica de investigación coherente con una concepción dialéctica de la realidad y del proceso de generación del conocimiento. Estos intentos cuestionan la intencionalidad política de la investigación social, las clases o grupos a que sirve y el tipo de sociedad a la que se contribuye a desarrollar". (pp. 78-79)

La Investigación Acción Participativa (IAP) se inscribe dentro de esta perspectiva. Uno de sus conceptos centrales es el de praxis, devenido de la teoría marxista y entendido como la acción transformadora y su correspondiente reflexión, "en contraposición al paradigma positivista que interpreta la praxis como simple manipulación tecnológica y control racional de los procesos naturales y sociales" (Fals Borda, 1978, pp. 223-224).

El involucramiento y compromiso con el contexto que se busca desde la IAP lleva al replanteamiento de la relación sujeto-objeto y, más aún, a la búsqueda de superación de las relaciones de dominación-dependencia, de las cuales forma parte el primer binomio. De la misma forma, la IAP se caracteriza por el reconocimiento de la "ciencia popular como algo válido" (Cetrulo et al., 1986, p. 20), sin partir de la base de que la única forma válida y generadora de conocimiento sea la ciencia, buscando caminos de encuentro entre el conocimiento académico y el conocimiento popular (Cetrulo et al., 1986, p. 22).

No obstante, y como fue señalado, el enunciado de "igualdad" no basta para poner a los conocimientos construidos por los diferentes actores en un mismo plano. El conocimiento tácito de los trabajadores puede o no coincidir con lo constatado a partir de la investigación científica y es necesario desmitificar creencias que muchas veces son portadas por los actores como conocimientos válidos (verdaderos). El sector académico aporta al proceso una metodología, un acumulado científico. No se parte de cero y no siempre el conocimiento construido desde el sector universitario está en línea con los conocimientos y opiniones construidos por los actores.

La IAP, entonces, constituyó la orientación general y estratégica del planteo metodológico y se construyó el proceso como un intento de coproducción de conocimiento entre universitarios y los trabajadores organizados. Ahora bien, integrar a los trabajadores a las distintas fases del proyecto no garantizaba la condición participativa. Es común en algunos colegas la confusión entre investigación participativa y empleo de técnicas cualitativas de recolección de datos, o entre lo que es extensión y la "consulta" a los actores. Los trabajadores podían ser incorporados solamente como informantes calificados o como destinatarios de una "de- 
volución" de resultados, sin que esto implicara un proceso de integración real, participación, producción conjunta, y mucho menos de extensión. Volvemos allí a los planteos del apartado anterior. El conocimiento no es neutral, se ubica en una relación social concreta. En este caso estábamos frente a un problema definido desde ciertos intereses, por lo tanto plantear una estrategia metodológica coherente obligaba a incorporar a los trabajadores desde las primeras etapas incluyendo la definición del problema.

Tanto la experiencia previa del equipo de trabajo como la bibliografía sobre vinculación universitaria con el sector productivo muestran que los problemas relacionados con la producción son planteados desde el sector empresarial pero enunciados con pretensión hegemónica como "los problemas de tal o cual sector" o como "problemas nacionales", haciendo abstracción de las relaciones sociales que tienen lugar en la producción, sin sesgo de intereses de clase. Enunciarlos desde la perspectiva de los trabajadores asalariados requiere hacer explícito lo que estamos asumiendo críticamente: situar los problemas definidos en la perspectiva del sector desde el cual se aborda. En este mismo sentido, es posible afirmar que generar instancias de participación y/o de dirección colectiva del proceso investigativo no es garantía de proceso de co-construcción de conocimiento ni de generación de conocimiento científico. Es en este punto, de acuerdo con el planteo de Etchebehere et al. (2018) puede afirmarse:

"Aparece así una suerte de dilema o de falsa oposición. Si el conocimiento desde la perspectiva adoptada no es neutral ante la realidad: ¿cómo es posible generar un conocimiento objetivo sobre lo real, despojado de parcialidades?... La realidad existe de forma independiente a la conciencia que los seres humanos tengan sobre ella. No es el objeto del capricho de la conciencia. De allí que exista la posibilidad de un conocimiento objetivo, esto es independiente a la voluntad de la conciencia sobre cómo debe ser lo real. No obstante, la conciencia humana aprende del mundo a medida que lo modifica. La conciencia exteriorizada, como acción humana, es creadora de la materialidad social y modifica permanentemente la realidad (Kosik, 1967)". (p. 22)

De esta manera, abordar el proceso de construcción de conocimiento como un doble proceso teórico y práctico supone que los conocimientos provenientes de los trabajadores y del equipo universitario son confrontados permanentemente en el camino teórico y metodológico. El saber universitario no se desprende, como el de los trabajadores, de la experiencia en el marco de relaciones sociales del complejo pesquero, y por ello deben ser puestos en diálogo.

La reflexión sobre los marcos conceptuales y la estrategia metodológica tiene que tener referentes concretos y coherentes en las herramientas de recolección y análisis de datos. Si el punto de partida era la hipótesis de "modelo productivo agotado", era necesario generar condiciones de posibilidad para verificarla en una serie de dimensiones. Fueron seleccionadas entonces algunas técnicas que en su planificación y ejecución involucraron directamente a los trabajadores. Se utilizaron seis conjuntos de técnicas en función de la vinculación entre el nivel de contacto de los trabajadores y el equipo universitario en el diseño y ejecución de las mismas, lo cual se presenta en el Cuadro 1. 


\begin{tabular}{|c|c|c|}
\hline $\begin{array}{l}\text { Actores que seleccionan } \\
\text { o diseñan las tareas }\end{array}$ & Ejecución & Tareas \\
\hline Universitarios & Universitarios & $\begin{array}{l}\text { Análisis de información secundaria: estadísticas de exportaciones, } \\
\text { evolución de capturas, recursos pesqueros. } \\
\text { Relevamiento de institucionalidad y normativa pesquera. }\end{array}$ \\
\hline Trabajadores & Trabajadores & $\begin{array}{l}\text { Registro de datos sobre esfuerzo pesquero. } \\
\text { Registros escritos y/o audiovisuales de operativa de buques. }\end{array}$ \\
\hline Universitarios & $\begin{array}{l}\text { Trabajadores } \\
\text { y universitarios }\end{array}$ & $\begin{array}{l}\text { Construcción del mapa sobre propiedad económica y grupos } \\
\text { empresariales. Descripción y análisis de formas de organización } \\
\text { del trabajo en pesca industrial, artesanal y plantas pesqueras. }\end{array}$ \\
\hline $\begin{array}{l}\text { Trabajadores } \\
\text { y universitarios }\end{array}$ & Trabajadores & $\begin{array}{l}\text { Estudio de buques pesqueros de captura de "pesquerías atípi- } \\
\text { cas". }\end{array}$ \\
\hline $\begin{array}{l}\text { Trabajadores } \\
\text { y universitarios }\end{array}$ & Universitarios & Elaboración y análisis de datos sobre la flota pesquera industrial. \\
\hline $\begin{array}{l}\text { Trabajadores } \\
\text { y universitarios }\end{array}$ & $\begin{array}{l}\text { Trabajadores } \\
\text { y universitarios }\end{array}$ & Observaciones de desembarques y recorridas por puertos. \\
\hline
\end{tabular}

Fuente: elaboración propia.

Entre las herramientas empleadas por el equipo universitario se empleó el análisis estadístico de información secundaria disponible en trabajos científicos, estadísticas pesqueras nacionales y datos recolectados. Como resultados de este análisis se pudieron determinar la evolución de las capturas, el esfuerzo pesquero, estimaciones de biomasa, análisis de desembarcos, comportamiento del comercio exterior, empleo y productividad.

Se obtuvo información de la bitácora de patrones de pesca de merluza y corvina (dos de las principales especies objetivo en las pesquerías de Uruguay) con datos de capturas, zonas de pesca, esfuerzo pesquero ${ }^{6}$ en horas y días de pesca. Esta información se consignó por mes y por año, lo cual permitió aproximarnos al estado de explotación de estas especies.

Fueron parte del material de campo fotografías y filmaciones realizadas por marineros sobre la operativa de los buques. Se hizo el mapeo de la propiedad económica del sector y los grupos empresariales, previa sistematización de información sobre empresas pesqueras, en la que los trabajadores participaron activamente (aportando su conocimiento sobre los vínculos entre empresas y en la construcción del mapeo de la propiedad del capital).

Una dimensión importante del complejo está constituida por la organización del trabajo. Para conocerla se diseñó y se llevó adelante una serie de talleres con trabajadores para reconstruir el proceso de trabajo identificando puntos críticos. Estos talleres fueron realizados con trabajadores tanto de la fase primaria (buques pesqueros y pesca artesanal) como de la fase secundaria (plantas procesadoras de pescado) y se complementaron con visitas a los buques durante la preparación de la salida de pesca o "marea" y visitas a puerto en los momentos de descarga de la captura. 
Otro instrumento empleado fue el análisis de dos casos. Para esto se seleccionaron dos buques de pesca industrial: uno que opera con red de arrastre de fondo dirigido a la captura de corvina (Micropogonias furnieri) y otro que opera dirigido a la captura de merluza (Merluccius hubbsi) con red de arrastre de fondo. Estas dos especies constituyen los recursos pesqueros más importante para la pesca en Uruguay (Norbis et al., 2006; Norbis y Galli, 2013) Este trabajo fue posible gracias a las relaciones establecidas con los trabajadores sindicalizados, quienes recabaron información confiable durante el viaje de pesca. El instrumento para la recolección de información fue un formulario de encuesta a los tripulantes. Esta encuesta la llevaron a cabo los propios trabajadores de cada barco, con previas instancias de formación y capacitación. De igual manera, se generó una bitácora de navegación donde registraron el día a día de la operativa a bordo. Por último, se contó con datos relativos a la operativa de los buques. Esta información permitió efectuar análisis sobre algunas pesquerías no tradicionales e identificar sus diferencias con las pesquerías tradicionales. ${ }^{7}$

El estudio de la normativa pesquera se realizó a través de la creación de un grupo de trabajo de integración mixta (universitarios y trabajadores). El objetivo de este grupo fue generar insumos para un proyecto de Ley de Recursos Hídricos que en ese momento estaba a consideración del Poder Legislativo nacional. Es decir que, durante el transcurso del proyecto, se combinaron los conocimientos en proceso de elaboración para ponerlos en acción en propuestas programáticas a plasmar en un marco legal.

Un desafío aparte lo constituyó la pesca artesanal como parte del complejo productivo. Para ello se implementaron recorridas en enclaves artesanales importantes de nuestro país (San Gregorio de Polanco, Constitución, Belén, La Paloma, Punta del Este, Piriápolis, costas de los departamentos de Montevideo y Canelones). En esas recorridas se relevó la dinámica del sector artesanal y sus vinculaciones con el resto del complejo, se generaron ámbitos de reflexión colectiva junto a cooperativas y pescadores artesanales de las zonas visitadas, y se recolectaron datos que permitieron comprender la dinámica particular de la pesca artesanal y sus enclaves, así como sus características sociales y económicas.

En el proceso fueron surgiendo otras formas de interacción entre el equipo universitario y los trabajadores, como fue la participación en sus asambleas para observar sus principales temas de discusión y agenda de problemas urgentes. En las instancias de asamblea se hizo además un espacio para ir presentando y poniendo en discusión los avances del proyecto. Asimismo, resultados parciales y primeras definiciones fueron publicados en el periódico sindical El Timón. Igualmente, el equipo universitario fue convocado por el sindicato a instancias de carácter político-institucional tales como un debate en el Parlamento nacional sobre modelos y políticas pesqueras (con participación de representantes del Poder Ejecutivo, parlamentarios, empresarios, universitarios, prensa y el PIT-CNT) ${ }^{8}$ y la implementación del denominado Consejo Sectorial Tripartito Pesquero en el marco de la promoción de una

7) En el caso uruguayo, se entiende por pesquería tradicional a aquella que tiene por especie objetivo a la merluza (Merluccius hubbsi) y la corvina rubia (Micropogonias furnieri) y que utiliza como artes de pesca redes de arrastre de fondo. Por pesquería no tradicional o atípica se entiende a aquella que no tiene como especies objetivo a las señaladas y que utiliza artes de pesca diferentes de las redes de arrastre de fondo. Estas definiciones están establecidas en la Ley No 19.175. Ver https://legislativo.parlamento.gub.uy/temporales/leytemp6446109.htm

8) PIT-CNT: Plenario Intersindical de Trabajadores-Convención Nacional de Trabajadores. Es la central única de los trabajadores organizados de Uruguay. 
estrategia nacional de desarrollo mediante el Gabinete Productivo en la órbita del MIEM. ${ }^{9}$

En tanto, una última herramienta fue la de la discusión prospectiva. La proyección de escenarios alternativos estuvo signada por la interacción en foros de debate con diferentes actores vinculados al sector. El rol del Estado en las políticas públicas relacionadas con la pesca, la creación de un ente testigo público y la importancia de desarrollar la investigación en el sector fueron los principales temas que se debatieron con el objetivo de buscar los cambios necesarios que surgieron en el contexto del proyecto.

\section{El análisis propositivo}

El análisis propositivo es un momento del proceso en el que se destaca el componente extensionista del proyecto, y está fuertemente ligado al proceso de "movilización del conocimiento" (Naidorf y Alonso, 2018; Naidorf, 2014). El concepto refiere a la vinculación de la producción del conocimiento científico con su uso. Señala Naidorf (2014):

"El concepto de movilización del conocimiento, entonces, asume diferentes perspectivas y definiciones: uso de la evidencia y del resultado de las investigaciones para la toma de decisiones en políticas públicas (Nutley et al., 2007); método o herramienta que facilita la traslación de resultados de la investigación a la acción (Bennet et al., 2007); esfuerzos por compartir resultados de investigación con posibles usuarios (Levin, 2011); acciones que permiten dejar el conocimiento listo para la acción y su intervención mediante interlocutores (Levesque, 2009)". (p. 83)

El análisis propositivo puede ser interpretado como el momento por excelencia de la puesta en acción del conocimiento. En este caso, antecedió, definió y superó el desarrollo de la investigación. Lo antecedió en el sentido de que la inquietud por cambiar el modelo pesquero nacional era previa incluso al contacto de los trabajadores con los universitarios del proyecto. La demanda expresada por los trabajadores ya contaba con una batería de medidas que estos consideraban necesarias para cambiar el modelo de desarrollo pesquero. Sin embargo, el proyecto les podía proporcionar un sustento técnico que, dada su posición subalterna en el sistema de relaciones sociales del complejo, no estaban en condiciones de generar por sí mismos. Y evidentemente trascendía el proyecto, pues era inherente al quehacer cotidiano de la vida sindical, el carácter propositivo de sus planteos programáticos y acciones. En tanto, desde el proyecto se contribuyó al desarrollo de propuestas. A partir de la información y el conocimiento generados se desarrollaron instancias de profundización, propuestas y debates en instancias abiertas a otros actores, así como el relevamiento de experiencias alternativas pasadas o actuales.

Se buscó construir un vínculo permanente entre el conocimiento producido y su apropiación por los actores.

Una parte de la investigación estuvo vinculada a la reconstrucción y seguimiento de experiencias antiguas y recientes en el sector pesquero, con el fin de poder identificar aquellas que se percibieron como alternativas al modelo imperante. De esta forma, se pudieron clasificar 
experiencias tanto en el ámbito del sector privado como en el ámbito estatal. Una vez identificadas estas experiencias alternativas se realizó un diagnóstico sobre las potencialidades, limitaciones y fracasos al momento de buscar transitar caminos distintos a los tradicionales. El resultado final permitió identificar problemas y lecturas compartidas, elementos conflictivos y diferencias en torno a los caminos que deberían orientar el desarrollo del sector en un futuro.

Los problemas que al comienzo de la investigación eran hipótesis fueron identificados claramente en el transcurso del trabajo: el modelo productivo agotado se manifestaba en una serie de síntomas-problemas sobre los cuales se necesitaba la elaboración de propuestas alternativas:

a. Concentración de la explotación pesquera en pocas especies.

b. Uso de artes de pesca depredadoras del fondo marino y hacia el conjunto de las especies de peces, la mayoría de las cuales eran descartadas.

c. Subordinación de la pesca artesanal a la lógica de explotación de la pesca industrial, con la consiguiente sobreexplotación del sector.

d. Disminución del empleo en las plantas pesqueras debido a la orientación casi exclusiva de la explotación al mercado exportador.

e. Flota pesquera obsoleta.

f. Organización del trabajo orientada a la permanente intensificación del trabajo, que no aprovechaba el conocimiento adquirido por los trabajadores ni permitía el desarrollo de una carrera profesional, con importantes dificultades en cuanto a seguridad a bordo y a salud laboral.

Se desarrollaron además talleres temáticos vinculados a los problemas que se iban detectando: situación de los recursos pesqueros, acuicultura, situación de la industria, organización y condiciones de trabajo en el sector. Estos talleres se complementaron con la realización de dos jornadas de discusión y debate a cargo de investigadores externos al proyecto con el propósito de debatir y reflexionar en torno a las propuestas realizadas por los trabajadores. La primera jornada incorporó elementos teóricos, metodológicos y de debate actual sobre "Estado y políticas públicas", los cuales permitieron potenciar la capacidad de análisis, reflexión y elaboración de propuestas sobre políticas pesqueras, con el objetivo de contribuir a la fase propositiva del proyecto. En la segunda jornada se analizaron elementos para el debate y la reflexión sobre el tema "Ente testigo", con el propósito de profundizar en el estudio de la propuesta de ente testigo pesquero, incorporando la evaluación de experiencias de décadas anteriores.

Al finalizar el trabajo de investigación se pasó a una instancia de trabajo colectiva. Los insumos empleados fueron las relatorías de los diferentes talleres y los documentos sindicales. La dinámica de trabajo consistió en la identificación de medidas tendientes a la transformación del sector. Para ello se tomaron tanto aquellas medidas propuestas previamente al desarrollo del proyecto por parte de los sindicatos integrantes de la intergremial marítima, como aquellas surgidas en el transcurso de mismo. Se analizó su viabilidad mediante la identificación de los actores involucrados y la afectación de sus intereses (tanto positiva como negativamente), la voluntad de los decisores y la viabilidad general de su implementación. A partir de ello se intentó diseñar una estrategia para el desarrollo de cada medida vinculándola al conjunto de propuestas. 
Las propuestas programáticas generales que fueron elaboradas se sintetizan en:

a. Orientar la explotación pesquera al conjunto de especies con potencial valor comercial y nutricional.

b. Indagar en la posibilidad de agregar valor a las capturas.

c. Considerar el mercado interno, fomentando el consumo de pescado.

d. Diversificar el uso de artes de pesca y realizar estudios de selectividad para conocer el efecto de las artes sobre los recursos.

e. Posibilitar procesos asociativos que fortalezcan al conjunto de pescadores artesanales

El conocimiento elaborado durante el proyecto fue movilizado en diferentes instancias y con distintos niveles de éxito:

a. Diseño de un emprendimiento pesquero cooperativo de trabajadores para la explotación de especies alternativas.

b. Propuesta y estudio de viabilidad de una planta pesquera de gestión cooperativa para la industrialización del producto obtenido por los pescadores artesanales.

c. Propuesta de mercado de concentración de capturas y productos pesqueros.

d. Elaboración de informes específicos para la participación de trabajadores en el Consejo Sectorial de la Pesca implementado por el Poder Ejecutivo en el anterior período de gobierno.

f. Inclusión de un artículo en la Ley de Compras públicas. ${ }^{10}$

g. Elaboración de insumos para el Departamento de Desarrollo Productivo del PIT-CNT.

Más allá de los dispositivos y propuestas concretas, desde el punto de vista de la extensión universitaria es posible identificar dos facetas diferentes pero complementarias:

por un lado, desde la concepción de extensión como una de las tres funciones sustantivas de la universidad, se hizo evidente la potencialidad de la integración de funciones. La extensión fue origen de un planteo investigativo pero fue también desarrollo y acción posterior. Es imposible pensar este proyecto de investigación sin un fuerte componente de extensión. Por otro lado, desde la concepción de la extensión como una herramienta que permite construir conocimiento desde un lugar diferente de la investigación, también puede señalarse que su utilidad quedó de manifiesto en este proceso.

Retomando lo señalado al inicio de este artículo, el proceso extensionista logró integrar el diálogo entre el actor universitario y los trabajadores a partir de la identificación de problemas cruciales que la contraparte no podía abordar por sí misma y con una vocación de crítica y propuesta de cambio al modelo actual de explotación pesquera.

\section{El período posinvestigación}

El período posterior a la investigación fue de continuidad de acciones conjuntas entre parte del equipo universitario original y los actores del sector. Se implementaron los llamados Nú-

10) Ley $N^{\circ} 19.685$. Establece, en su artículo $3^{\circ}$, un mecanismo de reserva mínimo de mercado del $30 \%$ para las compras centralizadas y del $100 \%$ para las no centralizadas de bienes alimenticios provenientes de "organizaciones habilitadas". Esos bienes alimenticios serán, entre otros, los productos provenientes de la pesca artesanal. 
cleos Interdisciplinarios de Estudios de la Pesca en el Uruguay (NIEPU) ${ }^{11}$ para trabajar sobre diferentes problemas concretos. Estos núcleos tuvieron lugar entre 2013 y 2019. Actualmente, algunos miembros del equipo se encuentran abocados al trabajo con los pescadores artesanales. El tema-problema que convocó al proyecto original sigue vigente y no se redujo a la realización del proyecto de investigación. Los años transcurridos permiten aventurar algunas reflexiones sobre el trabajo en conjunto universidad-sociedad y especialmente sobre algunas debilidades.

Con relación al vínculo universidad-sociedad, es importante resaltar que la conjunción de intereses comunes y la perspectiva superadora de nudos críticos que permitirían organizaciones más justas y con lugares más dignos para los sectores subalternos supera la vinculación. En la medida en que el Estado no forma parte mediante políticas públicas que permitan implementar las propuestas emanadas de estos proyectos participativos, las soluciones permanecerán en el ámbito de la enunciación o en acciones parciales y acotadas. Las propuestas señaladas no fueron oídas o tomadas en cuenta como objeto ya no de implementación sino de discusión. Las mayores medidas tomadas desde la decisión pública fueron la de un subsidio al combustible de los buques y un llamado a propuestas de reconversión de la flota pesquera que finalmente no prosperó. En ambos casos el problema de agotamiento del modelo actual del complejo productivo se agravó.

Lo señalado no quita validez a los proyectos que incluyen la integración de las funciones sustantivas de la universidad, pero permite afirmar su insuficiencia en términos sociales más generales en la medida en que no se aúnen los esfuerzos para cambiar realidades adversas por parte de los actores y los organismos públicos que pueden tomar decisiones. Al respecto, puede señalarse que los procesos de extensión que involucran grandes sectores de actividad, o núcleos grandes de población, tienen mayores dificultades para incidir en la realidad con vocación de cambio que aquellas intervenciones centradas en pequeñas comunidades o territorios acotados. En este caso, en el sector pesquero la influencia del proceso de extensión e investigación, frente a la ausencia de interlocutores de políticas públicas, quedó reducida a experiencias puntuales y a lo testimonial.

Sin embargo, la experiencia es altamente valiosa desde el punto de vista de la construcción de fortalezas en la población de trabajadores. Estos pudieron poner en valor su conocimiento, construir herramientas, relevar, discutir y apropiarse de los conocimientos que tenían y de los que en el transcurso del proyecto se fueron generando. El empoderamiento cognitivo de un sector subalterno es un resultado positivo que vale la pena considerar en su potencialidad de cambio, y en este proceso la extensión universitaria jugó un rol que consideramos relevante.

\section{Conclusiones}

El proceso del que se da cuenta es significativo como modalidad de vinculación entre la universidad y la sociedad y específicamente con el sector productivo. Se incorporaron actores

11) Los núcleos interdisciplinarios son espacios de investigación, enseñanza y extensión sobre un problema definido y abordado desde la interdisciplina. Son creados en el marco del Espacio Interdisciplinario de la Universidad de la República, unidad académica central que realiza, entre otras actividades, llamados concursables para el financiamiento de los núcleos por dos años. 
que hasta ahora estaban ausentes de la producción académica y fue posible unir disciplinas para pensar el sector en su globalidad.

La experiencia transcurrida plantea un desafío presente y futuro que tiene que ver con la posibilidad de construir agendas de investigación a partir de la extensión. A menudo esta función universitaria es vista como un subproducto de los procesos de enseñanza e investigación: la universidad se extendería, en la medida en que "reparte" el conocimiento generado en la sociedad. Esa concepción omite preguntarse sobre qué es lo que orienta a la universidad a investigar sobre determinados temas y no sobre otros. La experiencia descrita aporta un conjunto de elementos en clara contradicción con esta perspectiva. En primer lugar, porque el proceso de investigación surge en relación estrecha con un actor subordinado dentro de las relaciones de producción existentes y porque es a partir de este vínculo que surgen nuevos problemas y nuevos temas de investigación a futuro.

El contacto con sectores de la sociedad que en la generalidad de la investigación académica permanecen "invisibles" o reducidos a "objetos de estudio" puede plantear temas a incorporar en las agendas de investigación como problemas sociales pertinentes y sobre los cuales sus agentes y/o actores tienen cosas para decir y aportar. Evidentemente no toda la agenda universitaria de investigación surgirá de la función de extensión, pero esta última puede aportar a la primera, enriqueciéndola y acercándola más a las necesidades sociales, especialmente a aquellas vinculadas con la generación de realidades más equitativas, justas y democráticas.

La continuidad de la investigación se hizo explícita en tesis de grado y posgrado en el tema, así como en gran parte del equipo docente en el tema, profundizando en algunas de las líneas.

En lo relativo a la construcción de conocimiento conjunto, en este proceso fue difícil de separar cuánto de este proceso correspondía a la investigación y cuánto a la extensión. En cualquier caso se reforzó la idea de la creación de conocimiento situado, con una perspectiva determinada respecto de los problemas identificados.

Como fue dicho, los trabajadores vieron fortalecida su capacidad de identificar problemas, consolidar y/o confirmar conocimientos previos originados en el saber tácito, hacerse de bases científicas que sustenten y legitimen sus planteos programáticos, la posibilidad de identificar demandas e interlocutores.

Sin embargo, quizá sea más importante aún el hecho de que en el transcurso de esta experiencia los trabajadores fueron un agente de formación hacia el equipo universitario y entre ellos. Sin embargo, una vez culminado el proceso de la investigación, el vínculo con el actor universitario es menos frecuente y es difícil sostener la experiencia "solos", usando todo el aprendizaje en la vida cotidiana sindical. De esta manera se hizo evidente la importancia de la función de extensión como un proceso de largo plazo.

Durante el desarrollo emergieron dificultades derivadas de la inexperiencia en este tipo de desafíos epistemológicos. Y en lo referente a la relación con los sindicatos, una dificultad inicial consistió en construir un marco de trabajo común entre ambas partes. En particular, las expectativas previas de uno y otro lado no siempre coincidieron y muchas veces se fundamentaron en nociones previas sobre las capacidades del otro. Generar un acuerdo de trabajo siendo conscientes de los objetivos y posibilidades fue un proceso que difícilmente se haya saldado a pesar del tiempo transcurrido. 
Una dificultad a tener en cuenta en futuros proyectos de investigación deriva de los tiempos propios de la investigación académica con relación a los tiempos y urgencias de los actores sociales. Armonizar estos tiempos no es fácil puesto que el devenir de la realidad y los acontecimientos no es una variable sobre la que el equipo disponga a su antojo. Este aspecto no es secundario ya que incide en el nivel de oportunidad y pertinencia política del proceso de producción de conocimiento. Finalmente, este tipo de experiencias interpela al quehacer universitario desde el punto de vista de lo que Naidorf y Alonso (2018) denominan "los tres tiempos de la movilización del conocimiento": la definición de qué, por qué y para quién investigar (que pauta el "antes"), el cómo esa investigación se legitima y justifica dentro de las reglas de juego del campo académico en cuanto a parámetros de evaluación y ponderación por la comunidad (que pauta el "durante"), y la identificación de quién puede usar y poner en acción de forma útil el conocimiento generado (que pauta el "después" de la investigación). En este caso, estos tres tiempos fueron y son procesos complejos que se vinculan en forma permanente y que constituyen un desafío para la construcción de formas de hacer ciencia, enseñanza y extensión (en definitiva, de construir universidad) en un sentido que no siempre va en consonancia con las formas hegemónicas.

\section{Referencias bibliográficas}

Astori, D. y Buxedas, M. (1986). La pesca en el Uruguay: balance y perspectivas. CIEDUR-Ediciones de la Banda Oriental.

Buxedas M. (1994). Enfoques para el análisis: el sistema agropecuario y los complejos agroindustriales en la cuestión agraria en el Uruguay. FCU.

Cetrulo, R.; Fals Borda, O. y Rodríguez Brandao, C. (1986). Investigación Acción Participativa. Banda Oriental. Caussin, R.; Machorro-Ramos, F., Alfaro-Pérez, J.; Ramírez-Correa, P.; Painen-Aravena, G.; Melo, A. (2019). Transferencia de conocimiento tácito entre administrador y trabajador a través de la socialización: estudio en el sector minero chileno. Revista Ibérica de Sistemas e Tecnologias de Informação, (E17). http://www.scielo. mec.pt/scielo.php?script=sci_serial\&pid=1646-9895\&lng=pt\&nrm=iso

Etchebehere, C.; Galli, O.; Geymonat, J.; Mendy, M.; Morales, S. y Norbis, W. (2018). Análisis del complejo pesquero uruguayo: una experiencia de investigación y extensión con los trabajadores del mar. Colección Cassina. Extensión Libros Editora, CSEAM.

Fals Borda, O. (1978). Por la Praxis: El problema de cómo investigar la realidad para transformarla. Guadalupe. Fresán Orozco, M. (2004). La extensión universitaria y la Universidad Pública. Reencuentro, (39), 47-54. Universidad Autónoma Metropolitana Unidad Xochimilco.

Galli, O. (2005). Pesca sustentable y soberanía alimentaria en Uruguay. Un modelo para armar. Redes Amigos de la Tierra. Programa Uruguay Sustentable.

Galli, O. (2007). La pesca en el Uruguay: un modelo agotado. Redes Amigos de la Tierra. Programa Uruguay Sustentable.

Kosik, K. (1967). Dialéctica de lo concreto. Estudio sobre los problemas del hombre y del mundo. Grijalbo.

Mendy, M.; Morales, S.; Migliaro, A.; Santos, C.; Geymonat, J. y Etchebehere, C. (2011). La sustentabilidad del complejo pesquero desde una perspectiva interdisciplinaria. SCEAM. UdelaR. Grupo interdisciplinario de estudios sobre la pesca. Espacio Interdisciplinario. https://www.academia.edu/1206272/

Mendy, M.; Morales, S.; Geymonat, J.; Etchebehere, C.; Galli, O.; Norbis, W. y González, V. (2013). El proceso de construcción de la interdisciplina a partir de una investigación sobre el complejo pesquero uruguayo. 
En Fernandez, V.; Lorieto, A.; Repetto, A.; Nienni, B. y Von Sanden, C. (Eds.), En Clave_Inter: Procesos, contextos y resultados del trabajo interdisciplinario (pp. 121-127). Actas del ciclo En Clave de Inter del Espacio Interdisciplinario - UdelaR.

Mendy, M. y Marrero, N. (2020). Ciencia, capitalismo y coproducción de conocimiento. Cuadernos abiertos de Crítica y Coproducción. Las ciencias interrogadas. Fundamentos para una praxis científico-tecnológica transformadora. Grupo de Trabajo CLACSO "Prácticas emancipatorias y metodologías descolonizadoras transformadoras" http://biblioteca.clacso.org/clacso/gt/20200609051209/

Naidorf, J. y Alonso, M. (2018). La movilización del conocimiento en tres tiempos. Revista Lusófona de Educação, 39(208), 81-95.

Naidorf, J. (2014). Knowledge utility: from social relevance to knowledge mobilization. Education Policy Analysis Archive, 22(89). http//dx.doi.org/10.14507/epaa.v22n89.2014

Norbis, W.; Paesch, L. y Galli, O. (2006). Los recursos pesqueros de la costa de Uruguay: ambiente, biología y gestión. En Menafra, R.; Rodríguez-Gallego, L.; Scarabino, F. y Conde, D. (Eds.), Bases para la conservación y el manejo de la costa uruguaya (pp.197-209). Vida Silvestre.

Norbis, W. y Galli, O. (2013). Spatial cooccurrence of two sciaenid species (Micropogonias fuernieri and Cynocion guatucupa) subject to fishing in the Río de la Plata and oceanic coast of Uruguay: ecological or technological interdependence? Boletim do Instituto de Pesca, 2(39), 137-148.

Polanyi, M. (1966). The Tacit dimension. Doubleday \& Company Inc. Garden City.

Scarlatto, G. (1994). Notas sobre el papel del complejo arrocero en el desarrollo nacional. CIEDUR.

Tünnermann Bernheim, C. (2000). El nuevo concepto de extensión universitaria y difusión cultural y su relación con las políticas de desarrollo cultural en América Latina. Anuario de Estudios Centroamericanos, (4).

Sirvent, M. T. (2008). Educación de adultos: investigación, participación, desafíos y contradicciones. Miño y Dávila editores.

Vigorito, R. (1978). Criterios metodológicos para el estudio de complejos agroindustriales. ILET. 